\title{
Respuesta broncodilatadora del salbutamol administrado como aerosol presurizado mediante aerocámaras con válvulas o espaciadores no valvulados
}

\author{
CARLOS KOFMAN, ALEJANDRO TEPER, SANTIAGO VIDAURRETA, MARÍA TERESA KÖHLER
}

Centro respiratorio, Hospital de niños Ricardo Gutiérrez.

\begin{abstract}
Salbutamol bronchodilatatory response administered in pressurized aerosol through aerocameras with valves or not valvula spaciators

Introduction: Inhalation therapy is the most frequent route for administration of asthma treatment. The utility of spacers added to metered dose inhalers (MDI) is well established. However, non-valved spacers have recently been proposed as equally effective as the valved holding chambers, and more cost-effective. Clinical efficacy of non-valved spacers is not well established yet. Objective: To compare the bronchodilator response to albuterol administered by MDI with a valved holding chamber vs. a non-valved spacer. Patients and methods: In a experimental, prospective, randomized, simple blind, parallel group study, 34 asthmatic children from 6 to 16 years old with mild or moderate bronchial obstruction (force expiratory volume in the first second, $\mathrm{FEV}_{1} 50$ to $79 \%$ of predicted) without recent treatment whit bronchodilating agents were randomly assigned to be treated with $100 \mathrm{mg}$ of albuterol MDI (Ventolin ${ }^{\mathrm{TM}}$, MDI) either through a valved (Aerochamber ${ }^{\mathrm{tm}}$ ) oranon-valved $\left(\right.$ Aeromed $\left.^{\mathrm{tm}}\right)$ spacer. After 30 minutes, a bronchodilating response was determined through FEV, and FMF measurements. Results: 17 patients used the valved holding chamber (12 male, baseline $\mathrm{FEV}_{1} 67 \pm 10 \%$ ) and 17 the non-valved spacer (13 male, baseline $\mathrm{FEV}_{1} 67$ $\pm 6 \%$ ). Variation of $\mathrm{FEV}_{1}$ was $26 \pm 14 \%$ and $16 \pm 5 \%(\mathrm{p}=0,017)$ and variation of forced medium flow (FMF) was $92 \pm 48 \%$ and $58 \pm 29 \%(\mathrm{p}<0.018)$ for the valved holding chamber and for the non-valved spacer, respectively (ANOVA test). Conclusion: Albuterol administered by MDI through valved spacer produced a greater bronchodilator response than through non-valved spacer in asthmatic children

(Key words: spacers, bronchodilator response, asthma).
\end{abstract}

\section{RESUMEN}

Introducción: Los medicamentos para tratar asma utilizan fundamentalmente la vía inhalatoria. La utilidad 
de los espaciadores con válvulas (aerocámaras) está demostrada; sin embargo, recientemente se ha propuesto como una alternativa más económica el uso de espaciadores sin válvulas. La eficacia clínica de éstos no se ha demostrado hasta el momento. Objetivo: Comparar la respuesta broncodilatadora de un inhalador de dosis medida (IDM) de salbutamol administrado a través de una aerocámara contra un espaciador sin válvulas. Población, material y métodos: Estudio experimental, prospectivo, aleatorizado, simple ciego, de grupos paralelos. Se incluyeron 34 pacientes (6-16 años) con asma, que presentaban obstrucción bronquial leve o moderada (volumen respiratorio forzado, entre 50\% y $79 \%$ del valor predictivo), sin haber recibido broncodilatadores recientemente. Luego de una espirometría inicial se asignaron para recibir $100 \mathrm{mg}$ de salbutamol (aerosol presurizado) mediante una aerocámara con válvulas (Aerochamber ${ }^{\mathrm{mr}}$ ) o un espaciador sin válvulas (Aeromed ${ }^{\mathrm{mr}}$ ). Se determinó la respuesta broncodilatadora del volumen respiratorio forzado y del FMF a los 30 minutos. Resultados: En el grupo tratado con aerocámara ingresaron 17 pacientes (12 varones; volumen respiratorio forzado basal $67 \pm 10 \%$ ), y en el grupo con espaciador sin válvulas ingresaron 17 pacientes (13 varones, volumen respiratorio forzado basal $67 \pm 6 \%$ ). La variación del $\mathrm{FEV}_{1}$ fue $26 \pm 14 \%$ contra $16 \pm 5 \%(\mathrm{p}=0,017)$ y la variación del FMF fue $92 \pm 48 \%$ contra $58 \pm 29 \%(\mathrm{p}=0,018)$, para aerocámaras y espaciadores sin válvulas respectivamente (ANOVA). Conclusión: El aerosol presurizado de salbutamol administrado a través de aerocámaras con válvulas produce una respuesta broncodilatadora mayor que con espaciadores sin válvulas en niños con asma.

(Palabras clave: espaciadores, respuesta broncodilatadora, asma).

ESTE TRABAJO LO PUEDE ENCONTRAR EN EXTENSO EN WWW.SCIELO.CL 